\title{
9 A SAÚDE MENTAL EM ESTUDANTES DO ENSINO SUPERIOR. RELAÇÃo COM O GÉNERO, NÍVEL SOCIOECONÓMICO E OS COMPORTAMENTOS DE SAÚDE
}

\author{
Maria José Nogueira ${ }^{1}$; Carlos Sequeira ${ }^{2} \mid$
}

\section{RESUMO}

CONTEXTO: O nível de saúde mental (SM) influência o bem-estar e o sucesso académico dos estudantes do ensino superior (Chow, 2010), contudo esta problemática tem sido pouco estudada em Portugal. A literatura existente é pouco clara e, por vezes, contraditória no que respeita às variáveis psicossociais associadas positiva e/ou negativamente à SM dos estudantes do ensino superior (Eisenberg, et al., 2009).

OBJETIVO: (1) Caracterizar a SM dos estudantes do ensino superior; (2) explorar a relação entre a SM, variáveis sociodemográficas e comportamentos em saúde.

METODOLOGIA: Trata-se de um estudo descritivo, transversal e correlacional, e de uma amostra não probabilística, composta por estudantes do ensino superior do Distrito de Lisboa do $1^{\circ}$ e $2^{\circ}$ ano inscritos no $1^{\circ}$ ciclo de estudos. Os participantes deram consentimento informado escrito e completaram voluntariamente um inquérito online, que lhe foi enviado para o email do campus, durante outubro e novembro de 2014. Para recolher informação sociodemográfica, dos comportamentos de saúde e sobre a SM dos estudantes foram usados um questionário e o Inventário de Saúde Mental (ISM).

RESULTADOS: Globalmente, os resultados indicam níveis satisfatórios de SM, quer na dimensão positiva - Bem-Estar Psicológico como na negativa-Distress Psicológico. Contudo 93 participantes encontram-se com nível baixo de SM. As analises correlacionais mostram que as mulheres apresentam níveis de SM mais baixos que os homens, que à medida que o nível socioeconómico (NSE) aumenta, os níveis de SM melhoram. Os resultados também indicam que a níveis mais elevados de SM estão significativamente associados a participantes que praticam exercício físico regular e que dormem mais horas, em tempo aulas. Nesta amostra não se encontraram associações significativas entre o consumo de álcool e outras drogas e a SM dos estudantes.

CONCLUSÕES: Conclui-se que o género feminino e o NSE baixo são variáveis associadas negativamente à SM dos participantes. Enquanto a prática regular de exercício físico e dormir sete ou mais horas por dia se associaram positivamente à SM dos EES.

PALAVRAS-CHAVE: Saúde mental; estudantes do ensino superior; género; comportamentos de saúde

\section{RESUMEN}

\section{"Salud Mental en Estudiantes de Educación Superior. Relación con el género, el estatus socioeconómico y los comportamientos de salud"}

CONTEXTO: El nivel de influencia de la salud mental (SM) el bienestar y el éxito académico de los estudiantes en la educación superior (Chow, 2010), pero este tema ha sido poco estudiado en Portugal. La literatura existente no es clara ya por veces es contradictoria relativamente a las variables psicosociales asociados a la salud mental, positiva o negativa de los estudiantes de educación superior (Eisenberg, et al., 2009).

OBJETIVO: (1) caracterizar la SM de los estudiantes de educación superior; (2) explorar la relación entre las variables sociodemográficas, comportamientos de salud y la SM.

METODOLOGÍA: Se trata de un estudio descriptivo, transversal y correlacional, y una muestra no probabilística, integrado por estudiantes de educación superior en el Distrito de Lisboa del primer y segundo año, inscritos en el ler ciclo de estudios. Los participantes dieron su consentimiento informado por escrito y completaron voluntariamente una encuesta online, enviada para el correo electrónico del campus durante octubre y noviembre de 2014. Para recoger la información sociodemográfica, e sobre los comportamientos de salud y la salud mental de los estudiantes se usaron un breve cuestionario y el Inventario de Salud Mental (ISM).

RESULTADOS: En general, los resultados indican niveles satisfactorios de SM, ya sea en la dimensión positiva - Bienestar Psicológico como el la dimensión negativa - Distress Psicológico. Sin embargo, son 93 participantes con baja SM. Los análisis de correlación muestran que las mujeres tienen niveles de SM más bajos que los hombres. De igual modo, a medida que aumenta el nivel socioeconómico, los niveles de SM mejoran. Los resultados también indican que los niveles más altos de SM se asociaron significativamente con los participantes que se involucran en el ejercicio regular y dormir más horas en el tiempo de clase. En esta muestra no hubo asociaciones significativas entre el consumo de MS estudiantes de alcohol y otras drogas y.

CONCLUSIONES: Concluimos que el sexo femenino y el bajo nivel socioeconómico se asocian negativamente con la SM de los participantes. Mientras que el ejercicio físico regular y dormir más de siete o más horas al día se asoció positivamente con la SM de los participantes.

\section{DESCRIPTORES: Salud mental; estudiantes de educación supe- rior; género; comportamientos de salud}

\section{ABSTRACT \\ "The Mental Health of Higher Education Students: Relationship
with gender, socioeconomic status and health behaviours"}

BACKGROUND: The level of mental health (MH) affects the well-being and academic success of students of higher education (Chow, 2010). However, in Portugal, this issue has been rarely studied. The literature is unclear and sometimes contradictory regarding to positive and/or negatively associated psychosocial variables of higher education students (Eisenberg, et al., 2009).

AIM: (1) To characterize the MH of higher education students; (2) To explore the relationship between $\mathrm{MH}$ and sociodemographic and health behaviors variables.

METHODS: This is a descriptive, cross-sectional and correlational study, and a non-probabilistic sample, composed of students of higher education in the District of Lisbon of the 1st and 2nd year enrolled in the 1st cycle of studies. Participants gave written informed consent and, voluntarily completed an online survey, which was sent to campus email during October and November 2014. To collect sociodemographic information, health behaviors and students' $\mathrm{MH}$, were used a brief questionnaire and the Mental Health Inventory (ISM).

RESULTS: Overall, the results indicate satisfactory levels of $\mathrm{MH}$, both in the positive dimension - Psychological Well-Being and in the negative - Psychological Distress. However, 93 participants were found to have low MH level. Correlational analyzes show that women have lower levels of MH than men. Similarly, as the socioeconomic level increases, the levels of $\mathrm{MH}$ improve. The results, also, indicate that higher levels of $\mathrm{MH}$ are significantly associated with participants who exercise regularly and who sleep more than seven hours, during class period. In this sample, there were no significant associations between alcohol consumption and other drugs with students' $\mathrm{MH}$.

CONCLUSIONS: We conclude that the female gender and the low socioeconomic level are variables negatively associated with the $\mathrm{MH}$ of the participants. While regular practice of exercise and sleep seven or more hours per day were positively associated with $\mathrm{MH}$.

\section{KEYWORDS: Mental health; higher education students; gen- der; health behaviors}

Submetido em 27-01-2017

Aceite em 30-05-2017

1 PhD; Especialista em Enfermagem de Saúde Mental e Psiquiatria; ARSLVT, Av. Estados Unidos da América, 1749-096 Lisboa - Portugal, nogueira.mjc@gmail.com 2 PhD; Professor Coordenador da Escola Superior de Enfermagem do Porto, Porto - Portugal, carlossequeira@esenf.pt

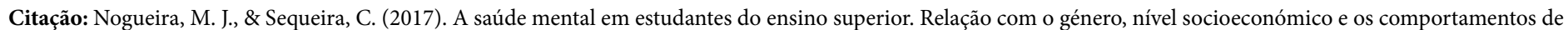
saúde. Revista Portuguesa de Enfermagem de Saúde Mental (Spe. 5), 51-56. 


\section{INTRODUÇÃO}

Os níveis de saúde mental (SM) em estudantes de ensino superior (EES) são inferiores quando comparados com os jovens não estudantes da mesma idade (Oliveira, 2011; Eisenberg, Hunt, \& Speer, 2013). Os níveis de SM influenciam a transição bem-sucedida (Meleis, 2011), o sucesso académico (Chow, 2010; Eisenberg, Golberston, \& Hunt, 2009) e o desempenho académico (Chow, 2010). A literatura é pouco clara e por vez contraditória no que respeita às variáveis associadas positiva e negativamente à saúde mental dos EES. A investigação indica que existe uma associação negativa entre a SM dos estudantes de ensino superior e o sexo feminino, o baixo nível socioeconómico (NSE) (Verger et al., 2009) e consumos de álcool e de substâncias psicoativas (Nemer, Fausto, Silva-Fonseca, Ciomei, \& Quintaes, 2013). Por oposição, são referidas associações positivas com a prática regular de exercício físico (Kenari et al., 2014), o número de horas de sono dormidas (Lopes et al., 2013), e o NSE elevado (Verger et al., 2009). Além disso, em Portugal pouco se conhece sobre o bem-estar ou o distress dos estudantes de ensino superior (Andrade, 2010) ou, sobre as variáveis assocadas, positiva ou negativamente. Deste modo o nosso objetivo foi caracterizar a SM dos estudantes de ensino superior e explorar as relações com variáveis sociodemográficas e comportamentos em saúde. Caracterizar a saúde mental dos EES e as variáveis associadas é importante para os enfermeiros. Esse conhecimento pode fornecer contributos relevantes, nomeadamente para fundamentar intervenções mais ajustadas às necessidades dos EES, no âmbito da promoção da saúde mental.

\section{METODOLOGIA}

Trata-se de um estudo com um design do tipo transversal, descritivo e correlacional e de uma amostra não probabilística de EES do Distrito de Lisboa, que frequentam o $1^{\circ}$ e $2^{\circ}$ ano do $1^{\circ}$ Ciclo de estudos. Os dados foram recolhidos durante o mês de outubro e novembro de 2015 e, ao longo dos procedimentos de recolha dos dados os aspetos formais e Éticos foram assegurados em conformidade com a Declaração de Helsínquia. Foi obtida a autorização escrita dos autores dos instrumentos usados no estudo. Os participantes deram o seu consentimento informado escrito e posteriormente completaram voluntariamente um inquérito anónimo online, contendo as variáveis em estudo, que lhe foi enviado para o email do campus. Previamente os participantes foram informados dos objetivos e implicações do estudo e da confidencialidade das suas respostas e foi ainda reforçado de que eram livres de desistir em qualquer altura da recolha dos dados. Cada participante no final do inquérito validou intencionalmente as sua respostas.

As variáveis em estudo incluem informação sociodemográfica - género, idade, NSE, estado civil, tipo de ensino, trabalho, bem como informação sobre os comportamentos de saúde - horas de sono dormidas, exercício físico, e consumos de álcool e outras drogas. Foi usado um breve questionário para recolher informação sobre estes itens. Para medir a variável saúde mental dos EES foi usado como instrumento de medida de autopreenchimento: o Inventário de Saúde Mental (ISM: Pais-Ribeiro, 2001). O ISM tem 38 itens distribuídos por uma dimensão negativa - Distress Psicológico, com 3 subescalas (ansiedade, depressão, perda de controlo emocional) e por uma dimensão positiva - Bem-Estar Psicológico, com 2 subescalas (afeto positivo e laços emocionais), e cota numa escala ordinal com 5 e 6 pontos.

O tratamento e análise estatística dos dados foi realizado utilizando o Software IBM $^{\circledast}$ SPSS $^{\circledast}$ Statistic Statistical Package for the Social Sciences for Windows, versão 22.0. Os dados foram analisados com recurso a técnicas de análise estatística descritiva, bivariada e inferencial (Marôco, 2011). Foram usados os testes $\mathrm{T}$ de Student para comparar dois grupos e a ANOVA quando se comparou três ou mais grupos. Recorreuse a testes Post hoc para destacar quais os grupos que diferem entre si. Sempre que se executou até cinco comparações recorreu-se ao Bonferroni. Foi assumido como nível de significância $p$ value $\leq 0,05$.

\section{RESULTADOS}

Participaram no estudo 560 estudantes, maioritariamente mulheres $(79,6 \%)$, com idade compreendida entre os 18 e os 24 anos e uma média de 19,6 anos $(\mathrm{DP}=1,6)$ e a quase totalidade são solteiros $(99,3 \%)$. Pertencem maioritariamente nível socioeconómico baixo. Metade coabita com os pais $(52,7 \%)$, mais de um quarto coabita com colegas/amigos (27,3\%), em menor percentagem com familiares (10,4\%), sozinho $4,1 \%$ ), com namorado(a)/companheiro(a)(3,2\%) ou outra situação (2,3\%). Aproximadamente um terço está deslocado de casa, e desses, a maioria vive com colegas/amigos $(67,4 \%)$. 
A maioria não trabalha e quem o faz trabalha entre uma e sessenta horas por semana $(M=21$ horas; $\mathrm{DP}=13,3)$. No que respeita à situação académica, $48,9 \%$ frequenta o primeiro ano e $51,1 \%$ segundo. A maioria frequenta o ensino público $(79,1 \%)$ sendo do ensino privado $17,4 \%$ e do Militar e Policial 3,5\% e, frequentam maioritariamente $(61,3 \%)$ cursos da área da saúde. Quanto aos comportamentos de saúde verificamos que $5,9 \%$ dormem uma media de oito ou mais horas de sono em tempo de aulas, $36,6 \%$ dorme entre sete a oito horas, $41,1 \%$ dorme entre seis a sete horas e $16,4 \%$ dorme entre cinco a seis horas por dia ou menos e, a maioria $(64,3 \%)$ considera que o tempo que dorme não é o suficiente. Mais de metade (52,5\%) pratica exercício físico, e de entre os que pratica exercício físico, a maioria $(46,3 \%)$ fá-lo duas a três vezes por semana, $28,2 \%$ pratica uma vez por semana, $20,1 \%$ pratica diariamente e $5,4 \%$ faz exercício físico uma vez por semana ou menos.

Relativamente à saúde mental dos participantes, os resultados revelaram uma média do ISM de 158,87 $(\mathrm{DP}=29,49)$, situando-se a média do Distress Psicológico em 107,41 (DP=19,41) e de Bem-Estar de $52,46(\mathrm{DP}=11,70)$, o que corresponde a níveis satisfatórios de SM.

A Tabela 1 mostra que a maior percentagem dos participantes se situa o nível Moderado, mas no nível Baixo encontram-se 93 estudantes.

Tabela 1 - Distribuição amostra por nível de saúde mental

\begin{tabular}{|l|l|l|}
\hline \multirow{2}{*}{ Nível de SM } & \multicolumn{2}{|l|}{ Amostra total $\mathrm{n}=560$} \\
\cline { 2 - 3 } & $\mathrm{n}$ & $\%$ \\
\hline Baixo & 93 & 16,6 \\
\hline Moderado & 379 & 67,7 \\
\hline Alto & 88 & 15,7 \\
\hline
\end{tabular}

No que respeita às comparações da saúde mental relativamente ao género, os resultados dos testes $\mathrm{T}$ apresentam-se na Tabela 2 , que revelam diferenças significativas $(\mathrm{t}(568)=3,30 ; \mathrm{p}=0,01)$ entre géneros em todas as subescalas do ISM, à exceção de laços Emocionais $(p>\alpha=0,05)$. Os participantes do género masculino tendem a apresentar melhor saúde mental que os do sexo feminino, tanto na dimensão positiva Bem-Estar Psicológico, como na dimensão negtiva- Distress Psicológico.
Tabela 2 - Comparação da saúde mental-ISM por género

\begin{tabular}{|c|c|c|c|c|}
\hline \multirow{3}{*}{ Subescalas ISM } & \multirow{2}{*}{$\begin{array}{c}\text { Amostra } \\
\text { Total } \\
\mathrm{n}=560\end{array}$} & \multicolumn{2}{|c|}{ Género } & \multirow{3}{*}{$\begin{array}{c}\text { Teste } \\
\mathrm{T}\end{array}$} \\
\hline & & $\begin{array}{c}\text { Feminino } \\
\mathrm{n}=446\end{array}$ & $\begin{array}{c}\text { Masculino } \\
\mathrm{n}=114\end{array}$ & \\
\hline & M & M & M & \\
\hline Distress Psicológico & 107,41 & 105,94 & 113,17 & $-3,59^{* *}$ \\
\hline Ansiedade & 41,64 & 40,98 & 44,21 & $-3,50^{* *}$ \\
\hline Depressão & 22,34 & 22,13 & 23,18 & $-2,25^{*}$ \\
\hline $\begin{array}{l}\text { Perda de Controlo } \\
\text { Emocional/ } \\
\text { Comportamental }\end{array}$ & 42,43 & 41,83 & 44,78 & $-3,87^{* *}$ \\
\hline Bem-Estar Psicológico & 52,46 & 51,87 & 54,77 & $2,37^{\star}$ \\
\hline Afeto Geral Positivo & 40,10 & 39,56 & 42,22 & $2,70^{\star \star}$ \\
\hline Laços Emocionais & 12,36 & 12,31 & 12,55 & ,74 \\
\hline ISM Global & 158,87 & 156,81 & 166,94 & $3,30^{* *}$ \\
\hline
\end{tabular}

${ }^{\star} \mathrm{P}<0,05 ;{ }^{* *} \mathrm{p}<0,01$

Quanto ao nível socioeconómico, a análise de variâncias revelou diferenças significativas (ANOVA; $\mathrm{F}(3)=4,24 ; \mathrm{p}=0,01)$ entre diferentes níveis socioeconómicos (Tabela 3). Constatou-se que os participantes de NSE- Elevado tendem a apresentar melhor saúde mental que os de NSE-Baixo (Bonferroni; $\mathrm{o}=0,011)$.

Tabela 3 - Comparação da saúde mental-ISM por NSE- ANOVA One-way

\begin{tabular}{|l|c|c|c|c|}
\hline \multirow{2}{*}{ Subescalas ISM } & \multicolumn{3}{|c|}{ Nível Socioeconómico } & \multirow{2}{*}{} \\
\cline { 2 - 4 } & $\begin{array}{c}\text { Baixo } \\
(\mathrm{n}=3)\end{array}$ & $\begin{array}{c}\text { Médio } \\
(\mathrm{n}=359)\end{array}$ & $\begin{array}{c}\text { Elevado } \\
(\mathrm{n}=18)\end{array}$ & $\mathrm{F}$ \\
\cline { 2 - 4 } & $\mathrm{M}$ & $\mathrm{M}$ & $\mathrm{M}$ & \\
\hline Distress Psicológico & 111,17 & 107,47 & 73,00 & $5,13^{\star *}$ \\
\hline Ansiedade & 43,17 & 41,65 & 29,33 & $3,16^{\star *}$ \\
\hline Depressão & 22,88 & 22,38 & 13,00 & $6,93^{\star *}$ \\
\hline $\begin{array}{l}\text { Perda de Controlo } \\
\text { Emocional/ } \\
\text { Comportamental }\end{array}$ & 41,11 & 42,44 & 29,67 & $5,06^{\star *}$ \\
\hline Bem-Estar Psicológico & 42,00 & 52,37 & 57,06 & $2,62^{\text {ns }}$ \\
\hline Afeto Geral Positivo & 32,33 & 40,02 & 44,00 & $2,59^{\text {ns }}$ \\
\hline Laços Emocionais & 9,67 & 12,35 & 13,06 & $1,38^{\text {ns }}$ \\
\hline ISM Global & 114,00 & 158,84 & 167,22 & $4,24^{\star *}$ \\
\hline$* \star$
\end{tabular}

${ }^{* *} \mathrm{p}<0,01 ;{ }^{\text {ns }}$ não significativo

No que respeita ao sono, a Tabela 4 mostra que existe uma correlação negativa e significativa entre a média de horas dormidas por dia em tempo de aulas e todas as subescalas do ISM $(\mathrm{p}<\boldsymbol{\alpha}=0,01)$. Os valores médios de saúde mental aumentam com o aumento do número de horas dormidas, sendo que o valor mais robusto se verifica com a subescala da ansiedade $(r=-, 23)$. 
Tabela 4 - Comparação da saúde mental por horas de sono dormidas

\begin{tabular}{|l|c|}
\hline Subescalas ISM & Horas de Sono ${ }^{(\mathrm{a})}$ rho \\
\hline Distress Psicológico &,$- 21^{\star *}$ \\
\hline Ansiedade &,$- 23^{\star *}$ \\
\hline Depressão &,$- 18^{\star *}$ \\
\hline $\begin{array}{l}\text { Perda de Controlo Emocional/ } \\
\text { Comportamental }\end{array}$ &,$- 16^{\star *}$ \\
\hline Bem-Estar Psicológico &, $20^{\star *}$ \\
\hline Afeto Geral Positivo &, $20^{\star *}$, \\
\hline Laços Emocionais &, $10^{\star}$ \\
\hline ISM Global &, $21^{\star *}$ \\
\hline
\end{tabular}

${ }^{*} \mathrm{p}<0,05 ;{ }^{* *} \mathrm{p}<0,01$;

(a) média de horas que dorme por dia em tempo de aulas;

No que concerne ao exercício físico, verificou-se que existem diferenças significativas $(\mathrm{t}(568)=3,54 ; \mathrm{p}=0,001)$ de saúde mental entre quem pratica e não pratica (Tabela 5). Quem pratica exercício físico tende a ter saúde mental significativamente superior aos que não praticam exercício físico, em todas as subescalas do ISM à exceção de Laços Emocionais.

Tabela 5 - Comparação da saúde mental por prática de exercício físico

\begin{tabular}{|l|c|c|c|}
\hline \multirow{2}{*}{ Subescalas ISM } & \multicolumn{2}{|c|}{ Exercício Físico } & \multirow{2}{*}{} \\
\cline { 2 - 3 } & $\begin{array}{c}\text { Pratica } \\
(\mathrm{n}=294)\end{array}$ & $\begin{array}{c}\text { Não pratica } \\
(\mathrm{n}=266)\end{array}$ & $\begin{array}{c}\text { Teste } \\
\mathrm{T}\end{array}$ \\
\cline { 2 - 3 } & $\mathrm{M}$ & $\mathrm{M}$ & \\
\hline Distress Psicológico & 104,60 & 109,95 & $3,26^{\star *}$ \\
\hline Ansiedade & 40,28 & 42,86 & $3,44^{\star *}$ \\
\hline Depressão & 21,77 & 22,87 & $2,95^{\star *}$ \\
\hline $\begin{array}{l}\text { Perda de Controlo Emocional/ } \\
\text { Comportamental }\end{array}$ & 41,55 & 43,22 & $2,68^{\star *}$ \\
\hline Bem-Estar Psicológico & 54,08 & 50,68 & $3,47^{\star *}$ \\
\hline Afeto Geral Positivo & 41,65 & 38,39 & $4,14^{\star *}$ \\
\hline Laços Emocionais & 12,43 & 12,29 &, $51^{\mathrm{Ns}}$ \\
\hline ISM Global & 163,03 & 154,28 & $3,54^{* *}$ \\
\hline
\end{tabular}

${ }^{* *} \mathrm{p}<0,01 ;{ }^{\text {ns }}$ não significativo

Quanto à relação entre o consumo de álcool e outras drogas e a saúde mental não se verificaram diferenças significativas a nenhum nível do ISM entre quem consumiu e não consumiu.

\section{DISCUSSÃO}

Com este estudo pretendeu-se caracterizar a SM dos EES e analisar as relações com variáveis psicossociais positivas e negativas, e nesse sentido acrescenta importante informação.
Verificamos que a amostra é constituída maioritariamente por participantes de sexo feminino, solteiros e com uma média de idade próxima de 20 anos. Este perfil está em linha com estudos prévios nesta população quer nacionais quer internacionais Eurostudent, 2012-2015 (Hauschildt, Gwosć, Netz, \& Mishra, 2015). Constatou-se que taxas de prevalência de consumos de álcool e drogas são ligeiramente inferiores aos valores reportados pela maioria dos estudos (Bayati, Beigi, \& Salehi, 2009; Ridner et al., 2015). A prática moderada de exercício físico/desporto pelos participantes é um dado positivo inesperado, contrariando a generalidade os estudos que indicam que os EES deixam de ter disponibilidade para praticar exercício físico (Ferrara, 2009).

Para a maioria dos participantes o número de horas que dormem por dia em tempo de aulas não é suficiente, e uma grande proporção dorme 5 a 6 horas ou menos horas por dia, resultados que confirmam valores de estudos prévios (Hershner \& Chervin, 2014). No que respeita à saúde mental dos participantes, globalmente, os resultados parecem indicar níveis de SM satisfatórios. Embora os valores de ISM sejam inferiores aos reportados em adultos jovens portugueses não estudantes (ISM=171,3) por Oliveira (2011), estão de acordo com resultados reportados em estudos prévios (Verger et al., 2009). Contudo, constatou-se que 93 participantes se encontram com um nível baixo de saúde mental.

As estatísticas bivariadas mostram diferenças no nível de SM entre géneros, as mulheres apresentam níveis significativos mais baixos de SM que os homens. Também o que respeita ao NSE dos participantes, este tem uma relação positiva com a saúde mental, à medida que o NSE aumenta os níveis de SM melhoram, tal como sugerido por vários autores (Bayati et al., 2009; Verger et al., 2009). As estatísticas também confirmam que os níveis mais elevados de SM estão associados aos participantes que praticam exercício físico com mais regularidade à semelhança de outros estudos com populações idênticas.

Também se constatou que aqueles que dormem mais horas de sono por dia em tempo aulas têm níveis de saúde mental mais elevados. Os nossos resultados estão em linha com outros estudos recentes nesta população (Ridner et al., 2015; Kenari, 2014). Não se apuraram associações entre o consumo de álcool e drogas e a SM dos participantes. 


\section{CONCLUSÃO}

Conclui-se que o género e o NSE- baixo são variáveis associadas negativamente à SM dos participantes. Enquanto a prática regular de exercício físico e dormir sete ou mais horas por dia está associada positivamente à SM dos EES.

\section{IMPLICAÇÕES PARA A PRÁTICA CLÍNICA}

Os resultados deste estudo são um importante contributo para a prática clínica, designadamente da enfermagem de saúde mental. Caracterizar a saúde mental dos EES e conhecer as variáveis, positivas e negativas associadas é essencial para adequar as terapêuticas de enfermagem. Pode igualmente ajudar a implementar estratégias de promoção de SM mais ajustadas às particularidades dos EES. Nomeadamente ao nível dos cuidados de saúde primários - saúde escolar, na preparação para a transição para o ensino superior, uma vez que os enfermeiros atuam na promoção da saúde e nos momentos antecipatórios das transições.

\section{REFERÊNCIAS BIBLIOGRÁFICAS}

Andrade, A., Duarte, P., \& Oliveira, L. (2010). Levantamento Nacional sobre o Uso de Álcool, Tabaco e Outras Drogas entre Universitários das 27 Capitais Brasileiras. GREA/IPQ-HCFMUSP. Brasília: SENAD: Secretaria Nacional de Políticas sobre Drogas.

Bayati, A., Beigi, A., \& Salehi, N. (2009). Depression prevalence and related factors in Iranian students. Pakistan Journal of Biological Sciences, 12(20), 13711375. doi: 10.3923/pjbs.2009.1371.1375

Chow, H. (2010). Predicting academic success and psychological wellness in a sample of Canadian undergraduate students. Electronic Journal of Research in Educational Psychology, 8(2), 473-496.

Eisenberg, D., Golberstein, E., \& Hunt, J. (2009). Mental Health and Academic Success in College. The B.E. Journal of Economic Analysis \& Policy, 9(1), 78-88.

Eisenberg, D., Hunt, J., \& Speer, N. (2013). Mental health in American colleges and universities: variation across student subgroups and across campuses. The Journal of Nervous Mental Disease, 201(1), 60-7. doi:10.1097/NMD.0b013e31827ab077.
Elias, A., Azevedo, V., \& Maia, A. (2009). Saúde e Rendimento Académico dos Estudantes da Universidade do Minho: Perceção de Áreas Problemáticas. In ESEP, Saúde e Qualidade de Vida em Análise (p. 349). Porto: ESEP- Núcleo de Investigação em Saúde e Qualidade de Vida.

Hauschildt, K., Gwosć, C., Netz, N., \& Mishra, S. (2015). Social and Economic Conditions of Student Life in Europe. EUROSTUDENT V 2012-2015. Bielefeld: Kristina Hauschildt. Retrieved from http://www. eurostudent.eu

Ferrara, C. (2009). The college experience: Physical activity, nutrition, and implications for intervention and future research. Journal of Exercise Physiology, 12(1), 23-35.

Gonçalves, A., Freitas, P., \& Sequeira, C. (2011). Comportamentos Suicidários em Estudantes do Ensino Superior: fatores de Risco e de Proteção. Millennium 40, $149-159$.

Kenari, M. (2014). Effect of Exercise on Mental Health in the Physical Dimension, Anxiety and Mental Disorder, Social Dysfunction and Depression. Advances in Applied Sociology, 4, 63-68. doi: 10.4236/aasoci.2014.43011

Lopes, E., Milheiro, I., \& Maia, A. (2013). Sleep quality in college students: a study about the contribution of lifestyle, academic performance and general well-being. Sleep Medicine, 14. doi: 10.1016/j. sleep.2013.11.437

Meleis, A. (2011). Toward the information network. Computer, 29(10), 59-67. doi: 10.1109/2.539723

Nemer, A. S. de A., Fausto, M. A., Silva-Fonseca, V. A. da Ciomei, M. H., \& Quintaes, K. D. (2013). Pattern of alcoholic beverage consumption and academic performance among college students. Revista de Psiquiatria Clínica, 40(2), 65-70. doi: 10.1590/S010160832013000200003

Oliveira, S. F. (2011). Saúde Mental e Consumo de Substâncias Psicoativas em Adultos na Comunidade. Escola Superior de Enfermagem do Porto. 
Pais-Ribeiro, J. (2001). Mental Health Inventory: Um Estudo de Adaptação à População Portuguesa. Psicologia, Saúde \& Doenças, 2 (1), 77-99.

Ridner, S. L., Newton, K. S., Staten, R. R., Crawford, T. N., \& Hall, L. A. (2015). Predictors of well-being among college students. Journal of American College Health, 64(2), 116-24. doi:o $\mathrm{rg} / 10.1080 / 07448481.2015 .1085057$
Verger, P., Combes, J., Kovess-Masfety, V., Choquet, M., Guagliardo, V., Rouillon, F., \& Peretti-Wattel, P. (2009). Psychological distress in first year university students: Socioeconomic and academic stressors, mastery and social support in young men and women. Social Psychiatry and Psychiatric Epidemiology, 44(8), 643-650. doi: 10.1007/s00127-008-0486-y

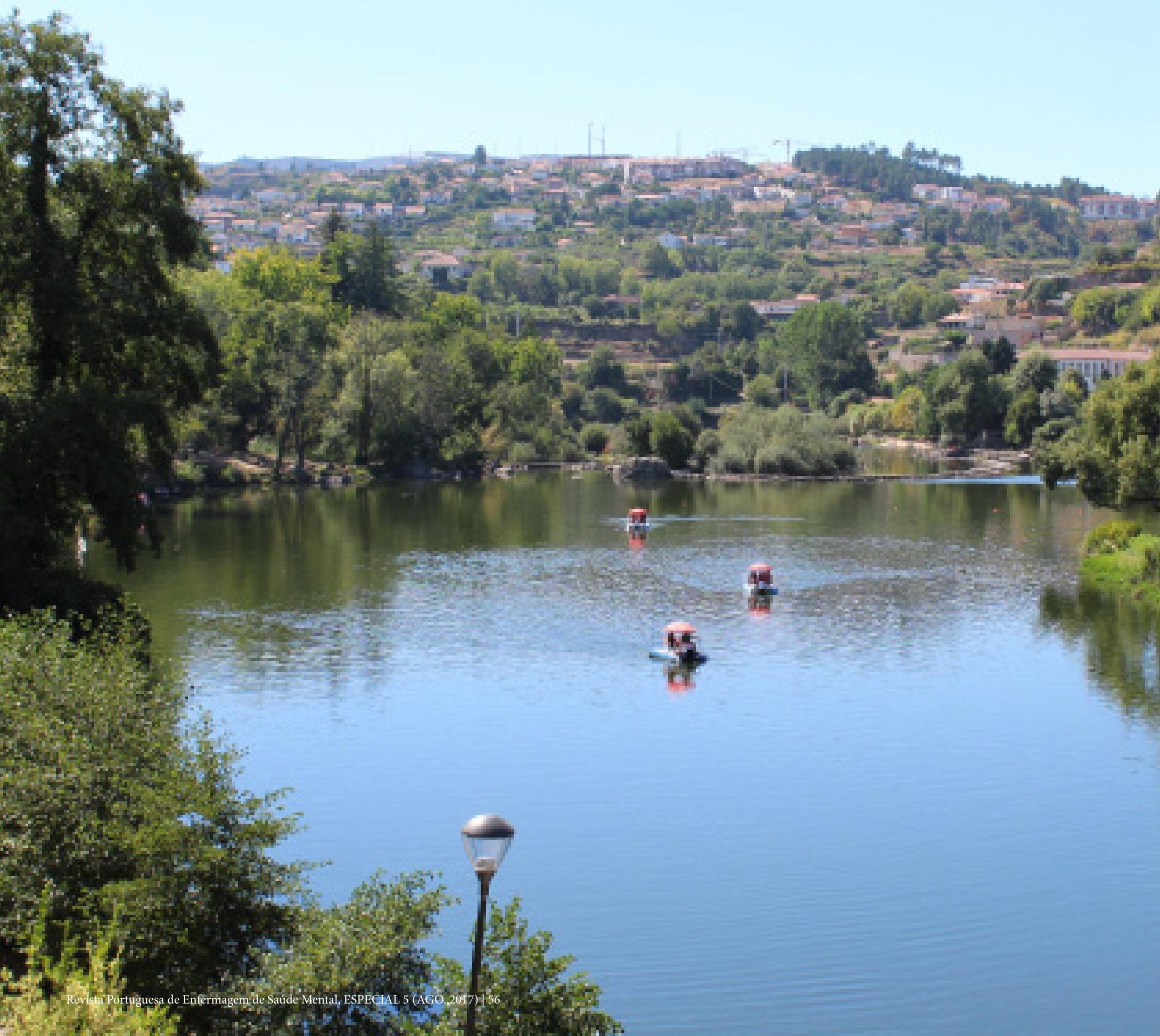

